Journal of Advanced Research in Fluid Mechanics and Thermal Sciences

Journal homepage: www.akademiabaru.com/arfmts.html ISSN: $2289-7879$

\title{
The Influence of Boundary Roughness on Rayleigh-Taylor Instability at the Interface of Superposed Couple-Stress Fluids
}

\author{
Krishna B Chavaraddi ${ }^{1}{ }^{*}$, Priya M Gouder ${ }^{2}$, Ramesh B Kudenatti ${ }^{3}$ \\ Department of Mathematics, S.S. Government First Grade College and P.G. Studies Center, Nargund-582207, Karnataka, India \\ Department of Mathematics, KLEs Dr. M. S. Sheshagiri CET, Udyambag, Belagavi-590008, Karnataka, India \\ Department of Mathematics, Central College Campus, Bangalore University, Bangalore-560001, India
}

\section{ARTICLE INFO}

\section{Article history:}

Received 9 March 2020

Received in revised form 29 April 2020

Accepted 4 May 2020

Available online 16 September 2020

\section{Keywords:}

RTI; boundary roughness; couple-stress

fluids; growth rate

\section{ABSTRACT}

The influence of boundary roughness on suppressing of Rayleigh-Taylor instability (RTI) at the interface between two superposed couples-stress fluids is investigated in the present paper. This is a theoretical study is based on fully developed approximations for deriving the growth rate of RTI at the interface between two fluids. Thus, it is found that the couple-stress parameter and boundary roughness have stabilization effect, whereas the buoyancy force (surface tension) has destabilize the system. Hence the study favors to control the growth rate of RTI.

Copyright @ 2020 PENERBIT AKADEMIA BARU - All rights reserved

\section{Introduction}

Rayleigh-Taylor instability (RTI) deals the instability between a less dense fluid below a high dense fluid has gained tremendous interest in both macrofluid and nano-fluid [1]. RTI plays a significant role in several natural processes extending from coastal upwelling, which supports to renew the nutrients near sea surface [2] to ignition of a supernova at the end of life of some stars [3]. The RTI is present in the formation of some astrophysical structures such as the supernova remnants in the Eagle and Crab-nebula [4], in the air bubble formation, in the blood of deep-sea divers [5] and in various industrial processes [6]. It is one of the main subjects of concern in ICF [7]. Presently RTI is being investigated in nano-composite fluid [8].

Along with these applications, RTI also occurs frequently in nature and hence it is required to study linear evolution of RTI, which involves precise mathematics based on normal mode analysis, producing exact solutions. However, it does not render an insight into the physical mechanisms underlying the instability [9]. Few studies describe RTI uses a physical argument based on energy balances, to make the mechanism of the instability more spontaneous may be due to Fermi

\footnotetext{
* Corresponding author.

E-mail address: ckrishna2002@yahoo.com
}

https://doi.org/10.37934/arfmts.75.2.110 
[10]. Nevertheless, the energy balancing between kinetic and potential energies requires the use of different masses for the calculation of each energy, which appears similar to a mathematical trick. Moreover, to describe more realistic situations involving viscous fluids and surface tension, such an argument cannot be extended easily $[11,12]$.

Bhatia [13] deliberated the impact of the viscosity on the stability of plane interface that separates two incompressible superposed conducting fluids in the presence of uniform magnetic field. He analyzed the stability of two highly viscous fluids with equal kinematic viscosity and different uniform densities. Shankar et al., [14] presented the work on stability of couple stress fluid flow through a horizontal porous layer and stability of natural convection in a vertical dielectric couple stress fluid layer in the presence of a horizontal ac electric field [15]. In the existence of a uniform horizontal magnetic field, Sunil [16] discusses the RTI of two superposed couples-stress fluids with uniform densities in a porous medium. Rudraiah and Chandrashekar [17] showed the effects of couple-stress fluid in controlling RTI at the interface between dense fluids. However, due to the importance of biomechanical and biomedical problems, the growth rate of RTI in non-Newtonian fluids are understood essentially (see Sharma and Sharma [18] and Rudraiah et al., [19]). The problems related to biomedical field viz., in synovial joints the synovial fluid is bounded by the porous nature of cartilages and in coronary artery diseases (CAD), the blood in the arteries is bounded by the porous nature of endothelium, the boundaries of the arteries. When joints degenerate the irregular shape of the interface between Cartilages and Synovial fluid deforms, producing surface instabilities In $C A D$ the cholesterol and other fat substances accumulate on the endothelium and form the plaques (i.e., the growth) on the endothelium. If the plaques reach a critical stage it blocks the flow of blood in the arteries and a cardiologist resort to by-pass or other devices like anagrams and so on. Coexistence of laminar, transitional and turbulent flow regimes is very common in blood flowing through arteries, air flows in human respiratory systems, and indoor airflow etc. Due to the complexity of flow physics involved, most Reynolds-averaged Navier-Stokes (RANS) turbulence models are not suitable for these flows because they are designed primarily for high Reynolds number turbulent flows [20]. In recent days, a high intensity laser is used to melt the plaque but it erodes the endothelium which causes the RTI. To avoid such side effects there is a need to understand the growth rate of RTI and suppresses the same. In recent days, a high intensity laser is used to melt the plaque but it erodes the endothelium which causes the RTI. To avoid such side effects, there is a need to understand the growth rate of RTI and suppresses the same. Shankar et al., [21] have contributed the applications of non-Newtonian fluid and the same researchers [22] were studied the MHD instability of pressure-driven flow of a non-Newtonian fluid. The linear and weakly nonlinear stability of a doubly diffusive electrically conducting non-Newtonian couple stress fluid layer in the presence of a uniform vertical magnetic field is investigated by Naveenkumar et al., [23].

During the degeneration, either in Synovial joints or in CAD the fluid deforms producing a spin field due to their micro rotation. Stokes [24] formulated the theory of couple-stress fluid. In the presence of uniform field, Agoor and Eldabe [25] discussed the RTI at the interface amongst two superposed couples-stress Casson fluids flowing in porous media. Chavaraddi et al., [26] studied the boundary roughness effect on RTI of a couple-stress fluid, which is bounded above by a clear fluid at the interface at $\mathrm{y}=\mathrm{h}$ and below by the roughness boundary at $\mathrm{y}=-\mathrm{h}$. Awati et al., [27] investigated the effect of boundary roughness on the nonlinear saturation of RTI in couple-stress fluid.

Recently, Gallaire and Brun [28] have discussed the fluid dynamic instabilities: theory and application to pattern forming in complex media in their review article. In this review article, they exemplify the use of stability analysis tools to rationalize pattern formation in complex media. Specifically, we focus on fluid flows, and show how the destabilization of their interface sets the 
blueprint of the patterns they eventually form. We review the potential use and limitations of the theoretical methods at the end, in terms of their applications to practical settings, e.g. as guidelines to design and fabricate the structures while harnessing instabilities. Rayleigh-Taylor instability in soft elastic layers is investigated by Riccobelli and Ciarletta [29]. The results of this work provide important guidelines for the design of novel soft systems with tunable shapes, with several applications in engineering sciences.

The main objective of this paper is to extend the work of Rudraiah and Chandrashekara [17] with the influence of boundary roughness on RTI in superposed couple-stress fluids. A simple model is constructed on the balance of forces and Stokes-lubrication approximations, which explains the physical mechanism that determines the instability of more complex situations. For viscous fluids like the couple-stress fluids, a simple approximation result in an explicit equation for growth rate of $\mathrm{RTI}$, which enable to understand the physical effects of boundary roughness, couple-stress parameter and buoyancy force (Bond number) on the instability evolution easily.

\section{Formulation of Problem}

The analysis is carried out for two superposed couple-stress fluids, which are incompressible, inviscous fluid film confined above by a fluid interface at $y=h$ and below by a horizontal solid boundary (rigid surface) at $y=-h$ with boundary roughness as depicted in Figure 1. Cartesian co-ordinate system aligned with the plane describes couple-stress fluid, $x$ points in horizontal direction and $y$ is vertical to it. The fluid properties of two layers are different; we use subscripts to distinguish them as $\rho_{1}$ and $\rho_{2}$ which denote densities in upper and lower couple-stress fluids respectively.

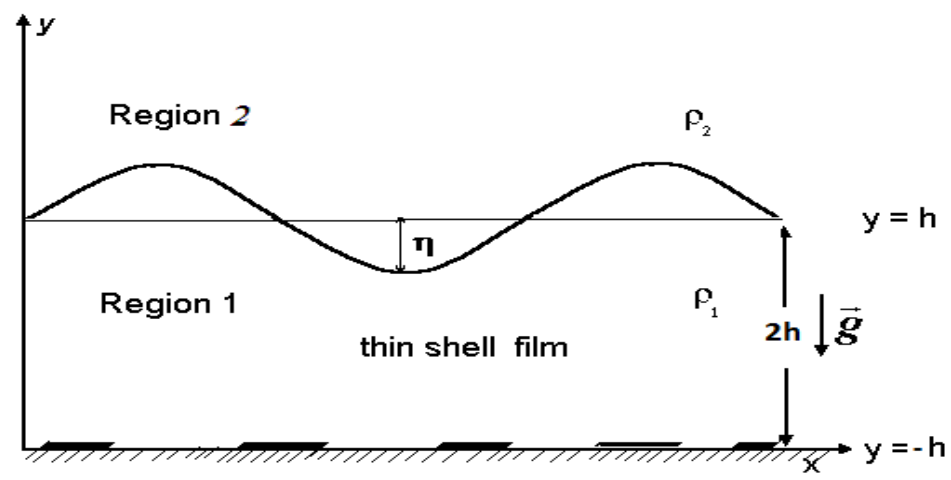

Fig. 1. Physical configuration

The system involves two semi-infinite inviscid, incompressible couple-stress fluids differentiated by an interface in the existence of surface roughness. Since gravity is present; the effective acceleration is in the positive $y$ - direction (upwards). Therefore, light fluid (region-1) thrusts on heavy fluid (region-2). As far as the interface amid fluids remains uniform, that is completely horizontal and perpendicular to effective acceleration. The light couple-stress fluid (Region-1) has adequate pressure to hold heavy fluid against the ceiling. However, small deviations are bound to occur at the interface. If the interface between the fluids is perfectly planar and in equilibrium, the fluid elements on both sides of the interface above and below must attain same pressure $p_{1}=p_{2}=p_{0}$ immediately. The rheological properties of physiological fluids reveal that viscosity varies nonlinearly with concentration exhibiting either shear thinning or shear thickening behaviour. Most of the existing literature has not considered micro motions, micro rotation and deformations. These are taken into account in this paper using a couple-stress fluid as a particular case of micropolar fluid theory [17]. The conservation of mass for an incompressible fluid is 
$\nabla \cdot \vec{q}=0$

The conservation of linear momentum

$\rho\left(\frac{\partial \vec{q}}{\partial t}+(\vec{q} \cdot \nabla) \vec{q}\right)=-\nabla p+\mu_{f} \nabla^{2} \vec{q}-\lambda \nabla^{4} \vec{q}$

These equations, satisfying the surface roughness and couple stress boundary conditions given in the subsequent section are solved.

\section{Solution of the Problem}

Here the RTI is developed using following combined lubrication and Stokes approximations [17].

i. The clear dense liquid is homogeneous and isotropic

ii. The thickness of film $h$ is much lesser than thickness $H$ of porous layer bounded above the film

i.e., $h \ll H$

iii. The Strouhal number $S$ is assumed to be considerably small.

iv. The surface elevation $\eta$ is assumed to be small compared to film thickness $h$.

i.e., $\eta<<h$

v. The fluid viscosity and thermal conductivity are assumed to be constant.

Also, the creeping flow approximation is used, which permits to neglect certain terms in the perturbation equation in obtaining linear equations for the interface elevation. Under the above approximations, the basic Eq. (1) and (2), for a two-dimensional flow lead to

$0=-\frac{\partial p}{\partial x}+\mu_{f} \frac{\partial^{2} u}{\partial y^{2}}-\lambda \frac{\partial^{4} u}{\partial y^{4}}$

$0=-\frac{\partial p}{\partial y}$

$0=\frac{\partial u}{\partial x}+\frac{\partial v}{\partial y}$

Using the following non-dimensional scales

$x^{*}=\frac{x}{h}, y^{*}=\frac{y}{h}, u^{*}=\frac{u}{\delta h^{2} / \mu_{f}}, v^{*}=\frac{v}{\delta h^{2} / \mu_{f}}, p *=\frac{p}{\delta h}, \beta^{*}=\frac{\beta}{h}$

The boundary and surface conditions the above equations are solved and the physical interpretation of the following boundary conditions as below 


\subsection{Surface-Roughness Condition}

This condition is a key factor in determining film quality and the physical properties of materials. It is defined as the shorter frequency of real surfaces relative to the troughs and quantified by the deviations in the direction of the normal vector of a real surface from its ideal form. If these deviations are large, the surface is rough; if they are small, the surface is smooth. Surface roughness is greatly affected by the microscopic asperity of the surface of each part.

$-\beta \frac{\partial u}{\partial y}=u$ at $y=-1$

\subsection{Interfacial Boundary Condition}

The interface condition (Neumann boundary condition) implies that no momentum can flow off the disk. These conditions describe reflecting phenomena on the boundary.

$$
\frac{\partial u}{\partial y}=0 \text { at } y=1
$$

\subsection{Couple-Stress Boundary Conditions}

These conditions are effective at points away from the boundary.

$\frac{\partial^{2} u}{\partial y^{2}}=0$ at $y= \pm 1$

\subsection{Dynamic Surface Condition}

The dynamic conditions at the interface consist of balance of the shear and normal stresses. Therefore, on the perturbed interface, in case of immiscible fluids, the capillary forces should be also taken into account. Here it is assumed that the flow in region-2 is static and flow in fluid layer is fully developed. We use the following normal stress or dynamic condition as

$$
p=-\delta \eta-\gamma \frac{\partial^{2} \eta}{\partial x^{2}} \text { at } y=1
$$

\subsection{Kinematic Surface Condition}

On the interface of two fluids, the kinematic boundary condition is considered in the form (continuity of normal velocity across the interface)

$v=\frac{\partial \eta}{\partial t}+u \frac{\partial \eta}{\partial x}$ at $y=1$

For linear case Eq. (12) reduces to

$v=\frac{\partial \eta}{\partial t}$ at $y=1$

By non-dimensionalizing the Eq. (5) to (7) and solving, we get, 
$\frac{\partial^{4} u}{\partial y^{4}}-\sigma^{2} \frac{\partial^{2} u}{\partial y^{2}}=-\sigma^{2} \frac{\partial p}{\partial x}$

By using the boundary conditions (8) to (13) in dimensionless form we obtain

$u=c_{1} \cosh \left(\sigma_{1} y\right)+c_{2} \sinh \left(\sigma_{1} y\right)+\left[\frac{y^{2}}{2}+1-A y-B\right] \frac{P}{\sigma^{2}{ }_{1}}$

where

$P=\frac{\partial p}{\partial x}, c_{1}=-\frac{P}{\sigma^{2}{ }_{1} \cosh \sigma_{1}}, c_{2}=0, A=\sigma_{1} P\left(\sigma^{2}{ }_{1}-\sigma_{1} \tanh \sigma_{1}\right)$

$B=\left[\left(\sigma^{2}{ }_{1}-\sigma_{1} \tanh \sigma_{1}\right)+2 \beta \sigma_{1} \tanh \sigma_{1}+\frac{\sigma^{2}{ }_{1}}{2}-2 \beta \sigma^{2}{ }_{1}\right] P$

\section{Dispersion Relation}

Eq. (7) is integrated, between the limits -1 to 1 and by using the condition (15), we obtain

$v(1)=\int_{-1}^{1} \frac{\partial u}{\partial x} d y$

$v(1)=\frac{\partial^{2} P}{\partial x^{2}} N$

where

$N=\frac{2}{\sigma^{2}{ }_{1}} \tanh \sigma_{1}\left(1-\sigma_{1}+2 \beta \sigma_{1}\right)-\frac{2}{\sigma^{2}{ }_{1}}-4 \beta+\frac{8}{3}$

Using Eq. (13) and (17) we get

$\frac{\partial \eta}{\partial t}=\left[\frac{\partial^{2} \eta}{\partial x^{2}}+\frac{1}{B_{1}} \frac{\partial^{4} \eta}{\partial x^{4}}\right] N$

By assuming the solution in the form $\eta=\eta(y) e^{i \ell x+n t}$, we get

$n=e^{2}\left[1-\frac{e^{2}}{B_{1}}\right] N$

The value of $n$ represented by Eq. (20) is calculated for several parameter values and the results are illustrated graphically in following Figure 2-4. 


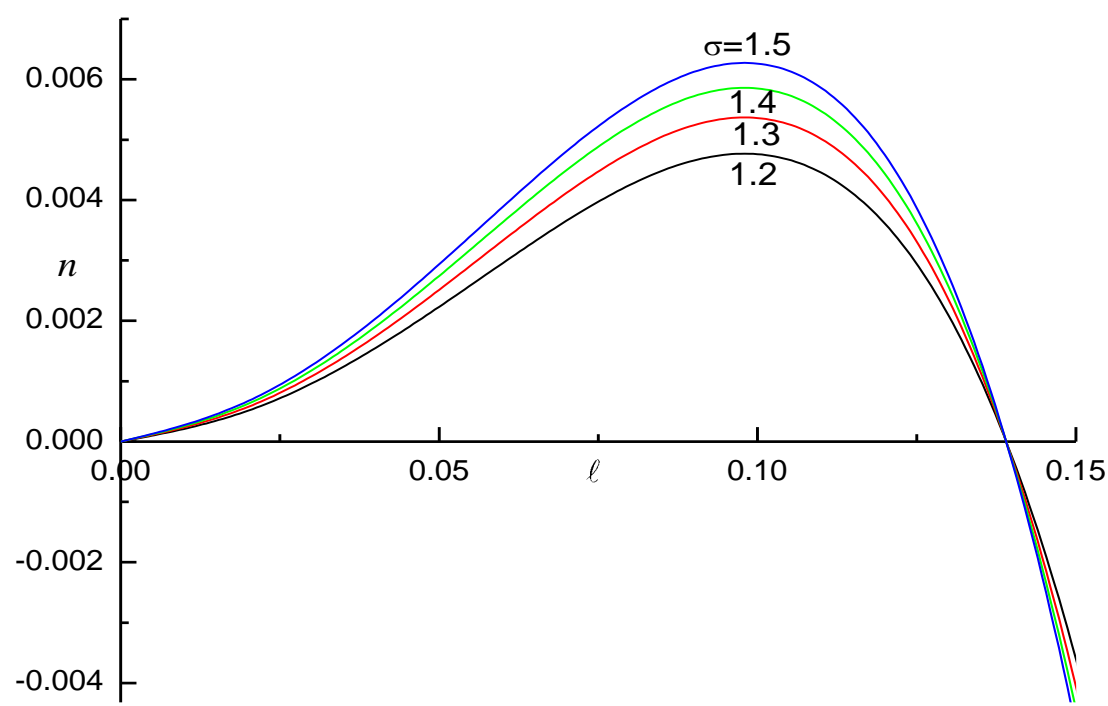

Fig. 2. Frequency versus wavenumber for different values of couplestress parameter $\sigma_{1}$ by fixing $B_{1}=0.02$ and $\beta=3.3 \times 10^{-2}$

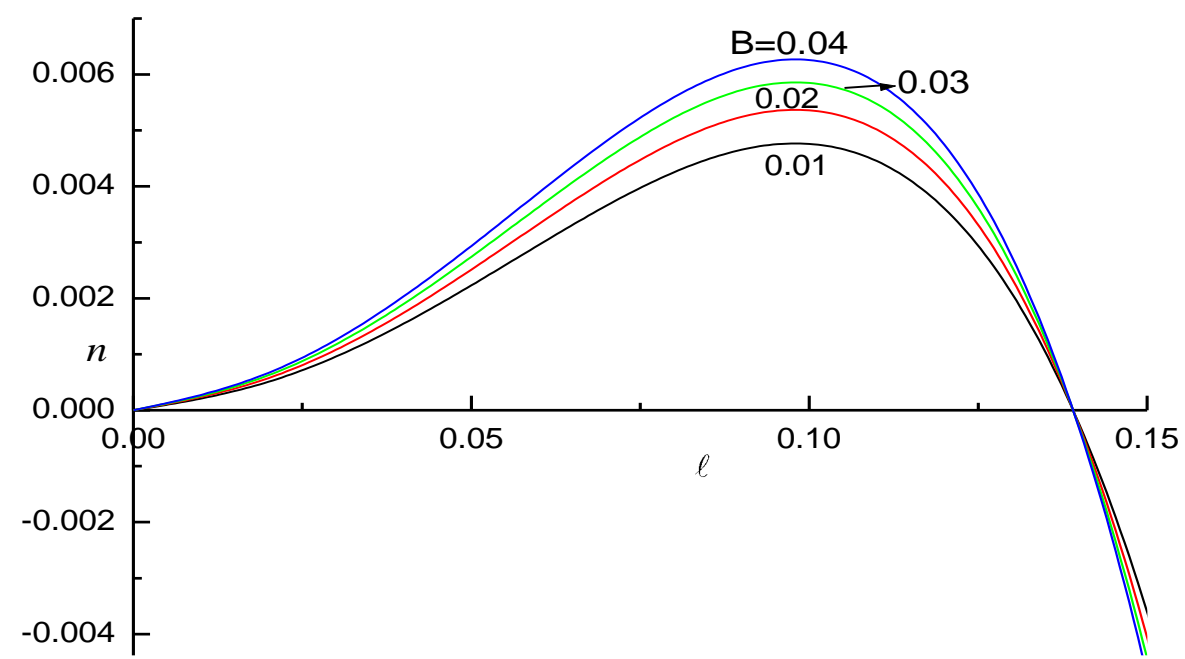

Fig. 3. Frequency $n$ versus wavenumber for different values of Bond number $B_{1}$ by fixing $\sigma_{1}=1.2$ and $\beta=3.3 \times 10^{-2}$

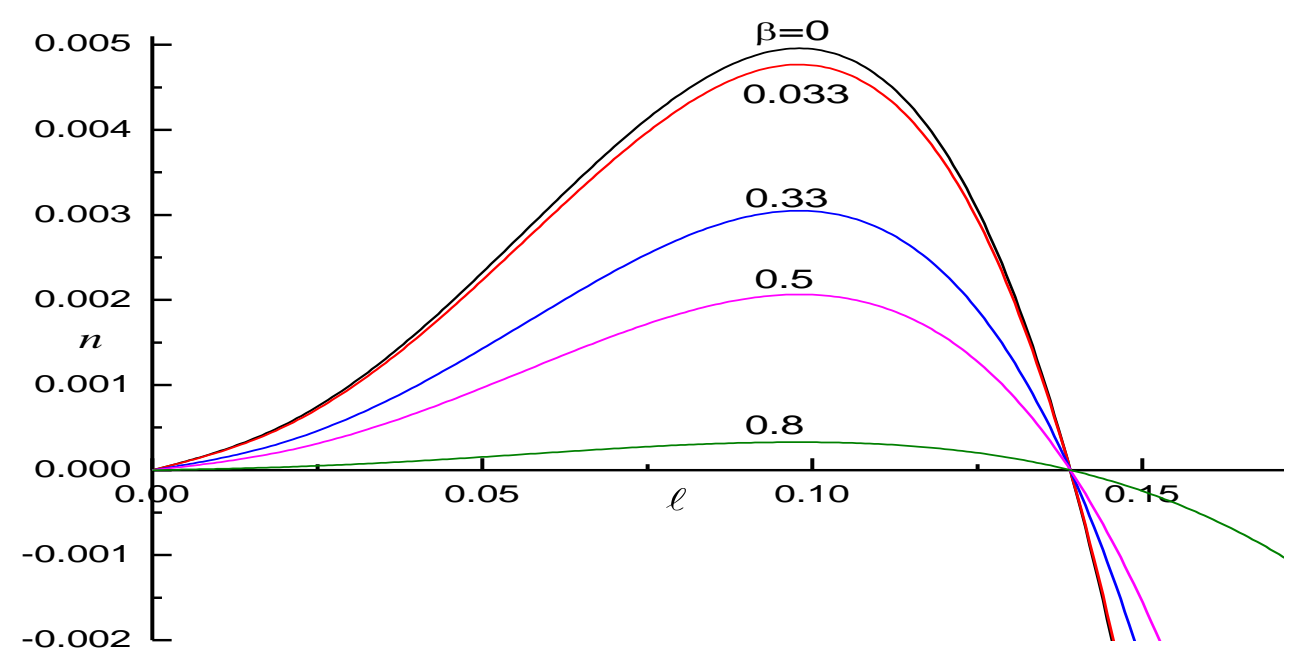

Fig. 4. Frequency versus wavenumber for different values of roughness parameter $\beta$ by fixing $\sigma_{1}=1.2$ and $B_{1}=0.02$ 


\section{Results and Discussion}

A theoretical study showing the boundary roughness effect on RTI at the interface of superposed couple-stress fluid layers are considered. This study is mathematically modelled by differential equations which governs the motion of fluids with suitable boundary and surface conditions. Under some assumptions, the resulting differential equation is solved. The influence of physical parameters involved in the problem for instance couple-stress parameter $\sigma_{1}$, Bond number $\mathrm{B}_{1}$ and roughness parameter $\beta$ on the region of stability are discussed numerically and depicted in graphs.

Figure 2 represents the influence of $\sigma_{1}$ on the frequency (growth rate) $n$. It is clear that increasing the couple-stress ratio results in slightly increasing the critical wave number and decreasing the maximum growth rate, this is because of the action of the body couples on the system. Thus, it has a stabilizing effect for the selected values of input parameters due to the increase in the couple-stress parameter.

Figure 3 depicts that $n$ decrease with increase in $B_{1}$ that governs the behavior of the flow and incorporates the surface tension $\gamma$, initial film thickness $h$ and substrate curvature. Below a critical value of $B_{1}$, the film is stable to perturbations with a thickness that decreases monotonically with time. For larger values of $B_{1}$, i.e., thicker films (or flatter substrates), the dynamic exhibit either transient perturbation growth followed by decay, or instability via drop formation. Finally, Figure 4 explains the dependence of $n$ on the roughness parameter $\beta$ cause the growth rate $n$. Therefore, it has negligible effect on reducing asymmetry of the system because of resistance offered by surface roughness in that process a part of kinetic energy is converted into potential energy. With this effect it concludes that the effect of surface roughness parameter $\beta$ is to stabilize the interface between the fluids though the couple-stress and buoyancy force (Bond number) have destabilizing effect on the system.

\section{Conclusions}

A theoretical study of the Rayleigh-Taylor instability (RTI) of the flow of couple-stress fluids is considered using linear stability analysis. This problem is modulated mathematically by a system of differential equations, which is governing the motion of the fluids with appropriate boundary conditions. The system of equations is solved under some assumptions to obtain the dispersion relation. The effects of the physical parameters of the problem such as couple stress parameter $\sigma_{1}$, roughness parameter $\beta$ and Bond number $B_{1}$ on the regions of stability are discussed numerically and depicted graphically. It is clear that the effect of roughness and couple-stress parameters are to stabilize the interface between two fluids, while the Bond number $\mathrm{B}_{1}$ has destabilizing effect.

\section{Acknowledgement}

The authors are grateful to the referees for their most valuable comments that improved the paper considerably. The author (PMG) would like to acknowledge the KLEs, Dr. MSSCET, Belgaum for their kind support. Also, the authors (KBC \& RBK) acknowledge the thanking UGC for the support and encouragement.

\section{References}

[1] Wakif, Abderrahim, Zoubair Boulahia, and Rachid Sehaqui. "A semi-analytical analysis of electro-thermohydrodynamic stability in dielectric nanofluids using Buongiorno's mathematical model together with more realistic boundary conditions." Results in Physics 9 (2018): 1438-1454.

https://doi.org/10.1016/i.rinp.2018.01.066 
[2] Cui, Anqing, and Robert L. Street. "Large-eddy simulation of coastal upwelling flow." Environmental Fluid Mechanics 4, no. 2 (2004): 197-223.

https://doi.org/10.1023/B:EFMC.0000016610.05554.0f

[3] Bychkov, V., and M. A. Liberman. "Hydrodynamic instabilities of the flame front in white dwarfs." Astronomy and Astrophysics 302 (1995): 727.

https://doi.org/10.1086/176257

[4] Ribeyre, X., V. T. Tikhonchuk, and S. Bouquet. "Compressible Rayleigh-Taylor instabilities in supernova remnants." Physics of Fluids 16, no. 12 (2004): 4661-4670.

https://doi.org/10.1063/1.1810182

[5] Racca, Robert A., and Clarence H. Annett. "Simple demonstration of Rayleigh-Taylor instability." AmJPh 53, no. 5 (1985): 484-486.

https://doi.org/10.1119/1.14206

[6] Andersson, H. I., and E. N. Dahl. "Gravity-driven flow of a viscoelastic liquid film along a vertical wall." Journal of Physics D: Applied Physics 32, no. 14 (1999): 1557.

https://doi.org/10.1088/0022-3727/32/14/301

[7] Piriz, A. R., J. Sanz, and L. F. Ibanez. "Rayleigh-Taylor instability of steady ablation fronts: the discontinuity model revisited." Physics of Plasmas 4, no. 4 (1997): 1117-1126.

https://doi.org/10.1063/1.872200

[8] Wakif, A., Z. Boulahia, A. Amine, I. L. Animasaun, M. I. Afridi, M. Qasimd, and R. Sehaqui. "Magneto-convection of alumina-water nanofluid within thin horizontal layers using the revised generalized Buongiorno's model." Frontiers in Heat and Mass Transfer (FHMT) 12 (2018).

https://doi.org/10.5098/hmt.12.3

[9] Chandrasekhar, S. "Hydrodynamic and Hydromagnetic Stability." Dover, New York, 1981

[10] Fermi, E. "Collected Papers, edited by E. Amaldi et al." (1962).

[11] Faber, Tom E. Fluid dynamics for physicists. Cambridge university press, 1995. https://doi.org/10.1017/CBO9780511806735

[12] Mikaelian, Karnig O. "Effect of viscosity on Rayleigh-Taylor and Richtmyer-Meshkov instabilities." Physical Review E 47, no. 1 (1993): 375.

https://doi.org/10.1103/PhysRevE.47.375

[13] Bhatia, P. K. "Rayleigh-Taylor instability of two viscous superposed conducting fluids." II Nuovo Cimento B (19711996) 19, no. 2 (1974): 161

[14] Shankar, B. M., I. S. Shivakumara, and Chiu-On Ng. "Stability of couple stress fluid flow through a horizontal porous layer." Journal of Porous Media 19, no. 5 (2016): 391-404. https://doi.org/10.1615/JPorMedia.v19.i5.20

[15] Shankar, B. M., Jai Kumar, and I. S. Shivakumara. "Stability of natural convection in a vertical dielectric couple stress fluid layer in the presence of a horizontal AC electric field." Applied Mathematical Modelling 40, no. 9-10 (2016): 5462-5481. https://doi.org/10.1016/i.apm.2016.01.005

[16] Sunil, Sharma RC, and R. S. Chandel. "On superposed couple-stress fluids in porous medium in hydromagnetics." $Z$. Naturforch., 57a (2002): 955-60. https://doi.org/10.1515/zna-2002-1208

[17] Rudraiah, N., and G. Chandrashekara. "Effects of couple stress on the growth rate of Rayleigh-Taylor instability at the interface in a finite thickness couple stress fluid." (2010): 83-89.

[18] Sharma, R. C., and K. C. Sharma. "Rayleigh-Taylor instability of two superposed conducting fluids in the presence of suspended particles." Acta Physica Academiae Scientiarum Hungaricae 43, no. 3-4 (1977): 251-258.

https://doi.org/10.1007/BF03159416

[19] Rudraiah, N., P. Sridharan, and S. Bhargava. "Rayleigh-Taylor instability in a finite thickness layer of a nonNewtonian fluid." Applied Mechanics and Engineering 5, no. 2 (2000): 315-327.

[20] Jiang, Jianbo, and Xinlei Wang. "Validation of Large Eddy Simulation in a Relaminarizing Boundary Layer Flow." CFD Letters 4, no. 1 (2012): 11-19.

[21] Shankar, B. M., Jai Kumar, I. S. Shivakumara, and K. R. Raghunatha. "Stability of natural convection in a vertical nonNewtonian fluid layer with an imposed magnetic field." Meccanica 53, no. 4-5 (2018): 773-786. https://doi.org/10.1007/s11012-017-0770-6

[22] Shankar, B. M., Jai Kumar, I. S. Shivakumara, and SB Naveen Kumar. "MHD instability of pressure-driven flow of a non-Newtonian fluid." SN Applied Sciences 1, no. 12 (2019): 1523. https://doi.org/10.1007/s42452-019-1557-2 
[23] Kumar, SB Naveen, I. S. Shivakumara, and B. M. Shankar. "Linear and Weakly Nonlinear Double-Diffusive Magnetoconvection in a Non-Newtonian Fluid Layer." Microgravity Science and Technology (2020): 1-18.

https://doi.org/10.1007/s12217-020-09781-4

[24] Stokes, Vijay Kumar. "Effects of couple stresses in fluids on hydromagnetic channel flows." The Physics of Fluids 11, no. 5 (1968): 1131-1133. https://doi.org/10.1063/1.1692056

[25] Agoor, B.M., and Eldabe, N.T.M. "RTI at the interface of superposed couplestress casson fluids flow in porous medium under the effect of magnetic field." J. Appl. Fluid Mechanics 7, no. 4 (2014): 573-580.

[26] Chavaraddi, Krishna B., Vishwanath B. Awati, and Priya M. Gouder. "Effect Boundary Roughness on Rayleigh-Taylor Instability of a Couple-Stress Fluid." Gen. Math. Notes 17, no. 2 (2013): 66-75.

[27] Awati, Vishwanath B., Krishna B. Chavaraddi, and Priya M. Gouder. "Effect of boundary roughness on nonlinear saturation of Rayleigh-Taylor instability in couple-stress fluid." Nonlinear Engineering 8, no. 1 (2019): 39-45. https://doi.org/10.1515/nleng-2018-0031

[28] Gallaire, François, and P-T. Brun. "Fluid dynamic instabilities: theory and application to pattern forming in complex media." Philosophical Transactions of the Royal Society A: Mathematical, Physical and Engineering Sciences 375, no. 2093 (2017): 20160155. https://doi.org/10.1098/rsta.2016.0155

[29] Riccobelli, Davide, and Pasquale Ciarletta. "Rayleigh-Taylor instability in soft elastic layers." Philosophical Transactions of the Royal Society A: Mathematical, Physical and Engineering Sciences 375, no. 2093 (2017): 20160421.

https://doi.org/10.1098/rsta.2016.0421 